\title{
Primer registro de Amorphinopsis atlantica Carvalho, Hadju, Mothes \& van Soest, 2004 (Familia: Halichondriidae) para un sistema lagunar del golfo de México
}

\section{First record of Amorphinopsis atlantica Carvalho, Hadju, Mothes \& van Soest, 2004 (Family: Halichondriidae) for a lagoon system in the Gulf of Mexico}

Vicencio de la Cruz-Francisco ${ }^{1 *}$, Jimmy Argüelles-Jiménez ${ }^{2}$, Salvador Rodríguez Muñoz ${ }^{1}$, Ramses Giovanni León Méndez ${ }^{1}$ y Aarón Duran López ${ }^{1}$

\begin{abstract}
RESUMEN
Se registra por primera vez la presencia de Amorphinopsis atlantica en un sistema lagunar del golfo de México. Esta esponja fue reportada en Brasil donde prefiere asentarse sobre costas rocosas y en estuarios. Las observaciones y recolecta de especímenes provienen de la laguna de Tampamachoco, ubicada al norte de Veracruz, México. Los ejemplares registrados se contemplaron como epibiontes en bancos ostrícolas de Isognomon alatus, donde destacaron por su coloración amarilla, y su forma incrustante ahí masiva con ramificaciones prolongadas. Presenta megascleras oxeas (331-774/7-16 $\mu \mathrm{m}$; largo/ancho) y estilos (138-205/5-9 $\mu \mathrm{m}$; largo/ ancho). Con base en los avistamientos realizados del 2015 a la fecha, se considera que $A$. atlantica es una especie establecida en el sistema lagunar. El presente registro aumenta el ámbito de distribución original de $A$. atlantica hacia las costas del golfo de México.
\end{abstract}

Palabras clave: esponjas, distribución, estuario, manglares, laguna de Tampamachoco, Amorphinopsis atlantica

\footnotetext{
ABSTRACT

Amorphinopsis atlantica is recorded for the first time in a lagoon system of the Gulf of Mexico. This sponge was reported in Brazil where it prefers to settle on rocky shores and estuaries. Specimens were observed and collected from the Tampamachoco lagoon in northern Veracruz, Mexico.

Facultad de Ciencias Biológicas y Agropecuarias, Universidad Veracruzana., Carr. Tuxpan-Tampico km 7.5, C.P. 92854, Tuxpan, Veracruz, México. ${ }^{2}$ Instituto de Ciencias Marinas y Pesquerías, Universidad Veracruzana, Hidalgo 617, Col. Río Jamapa, C.P.94290, Boca del Río, Veracruz, México.viccruz@uv.mx*, cayix24@gmail.com, b.m.salvador.rodriguez@gmail.com, giovannileon96@gmail.com, aa.dlpz0296@gmail.com
} 
Subjects observed were epibionts on Isognomon alatus oyster beds, standing out for their yellow coloration and massively encrusting form with prolonged branches. Megascleres both oxeas (331-774/7-16 $\mu \mathrm{m}$; length/width) and styles (138-205/5-9 $\mu \mathrm{m}$; length/width) were present. Based on the observations made from 2015 to date, A. atlantica is considered to be a species well established in the lagoon system. This record increases the original distribution range of $A$. atlantica towards the coasts of the Gulf of Mexico.

Keywords: sponges, distribution, estuary, mangroves, Tampamachoco lagoon, Amorphinopsis atlantica

\section{INTRODUCCIÓN}

El género Amorphinopsis está representado por 17 especies válidas (Van Soest et al. 2018), de las cuales, Amorphinopsis atlantica, es la única especie del género que se ha descrito para el océano Atlántico (Santos et al. 2018). El primer registro de esta especie se realizó en Brasil, donde habita en costas rocosas y estuarios (Carvalho et al. 2004). Ecosistemas similares ocurren a lo largo de las costas del golfo de México; no obstante, a la fecha no existen anotaciones previas de la presencia de Amorphinopsis. En la zona costera de Veracruz, alrededor de 70 especies de esponjas se han listado en inventarios taxonómicos principalmente para ecosistemas de arrecifes de coral (Gómez, 2002; 2007; González-Gándara et al. 2015; De la Cruz-Francisco y Bandala-Pérez, 2016; De la Cruz-Francisco \& González-González, 2016; De la Cruz-Francisco et al. 2016; 2017) y de intermareales rocosos (De la Cruz-Francisco et al. 2017).

Por lo anterior, el propósito del presente trabajo es dar a conocer el primer registro de $A$. atlantica en sistemas estuarinos del golfo de México, información que incrementa el conocimiento de la fauna de esponjas que se establece en la laguna de Tampamachoco, Veracruz, donde previamente se tiene el hallazgo de Clathria sp. y Haliclona manglaris como epibiontes en raíces de Rhizophora mangle (Lucas \& De la Cruz-Francisco, 2018).

\section{MATERIALES Y MÉTODOS}

Área de estudio. La laguna Tampamachoco se localiza en el municipio de Tuxpan, Veracruz, México (entre $21^{\circ} 02^{\prime} 10^{\prime \prime} \mathrm{N}, 97^{\circ} 22^{\prime}$ '50" W у $20^{\circ} 58^{\prime} 40^{\prime \prime} \mathrm{N}, 97^{\circ} 20^{\prime} 29^{\prime}$ ' W, Fig. 1). Es un cuerpo de agua somero de elevada turbidez (Contreras, 1983), de características meso-polihalinas donde la salinidad fluctúa entre 20 y 40 UPS y la temperatura superficial de $25 \mathrm{y}$ $30^{\circ} \mathrm{C}$ (Lara-Domínguez et al. 2011). A lo largo del año, presenta variaciones; por ejemplo, durante la primavera se observa un incremento de la salinidad que disminuye en otoño, mientras que la temperatura es mayor en verano y 
menor en invierno (López-Ortega et al. 2012). La laguna de Tampamachoco abarca un área de $15 \mathrm{~km}^{2}$, presenta aproximadamente $10.6 \mathrm{~km}$ de largo, $2.7 \mathrm{~km}$ de ancho y se encuentra separada del mar por una barrera arenosa.

Diseño de muestreo. Esponjas masivas de color amarillo, fueron recolectadas en bancos ostrícolas, al suroeste de la laguna Tampamachoco durante los meses de septiembre de 2015, abril de 2016 y noviembre de 2018. Dicha área, se caracteriza por ser somera ( $1 \mathrm{~m}$ de profundidad) y presentar sustrato fangoso con parches de Thalassia testudinum y Halodule wrightii. En cada muestreo se revisaron de 10 a 20 colonias de ostras (Isognomon alatus) para detectar incrustaciones de esponjas, las que fueron desprendidas de las valvas con el uso de una navaja y posteriormente fueron conservadas en alcohol etílico al 70\%. La identificación taxonómica se realizó con base en la coloración, forma, consistencia, reticulación esquelética y espículas,

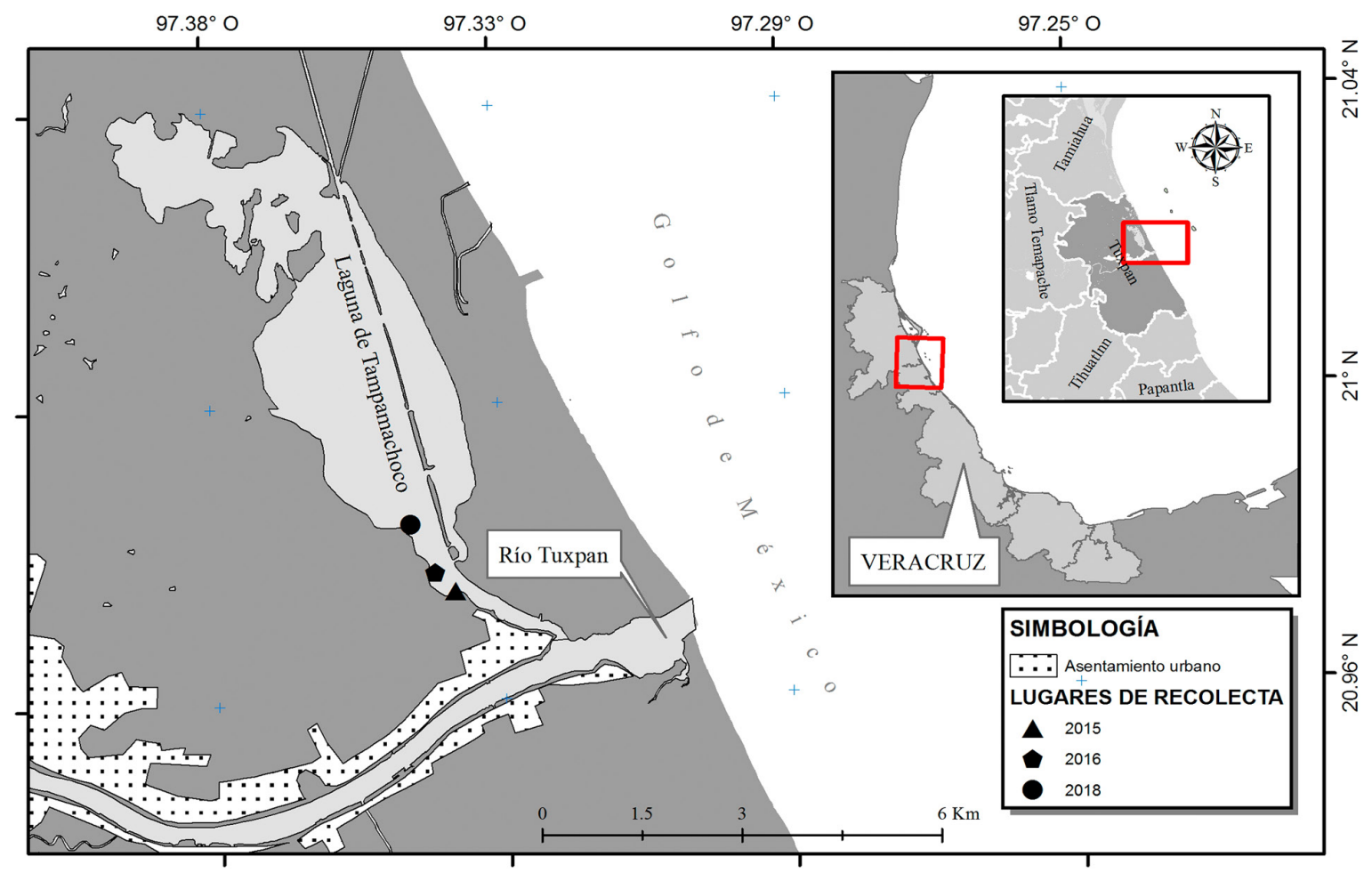

Fig. 1. Estaciones de observación y recolecta de Amorphinopsis atlantica, en la laguna de Tampamachoco, Tuxpan, Veracruz

Fig. 1. Observation and collection stations of Amorphinopsis atlantica in the Tampamachoco lagoon, Tuxpan, Veracruz 
atributos que se detallan en las publicaciones de Carvalho et al. (2004) y Santos et al. (2018). Todos los especímenes recolectados se depositaron en la colección biológica de la Facultad de Biología de la Universidad Veracruzana, Campus Tuxpan (FBUVP). Para cada ejemplar, se realizaron 30 mediciones de las espículas, las medidas que se registraron fueron: mínimo-promedio-largo máximo y mínimo-promedio-ancho máximo.

\section{RESULTADOS}

Descripción sistemática

Phylum: Porifera

Clase: Demospongiae

Subclase: Heteroscleromorpha

Orden: Suberitida Chombard \&

Boury-Esnault, 1999

Familia: Halichondriidae Gray, 1867 Amorphinopsis atlantica Carvalho, Hadju, Mothes \& van Soest, 2004

Figs. 2-3
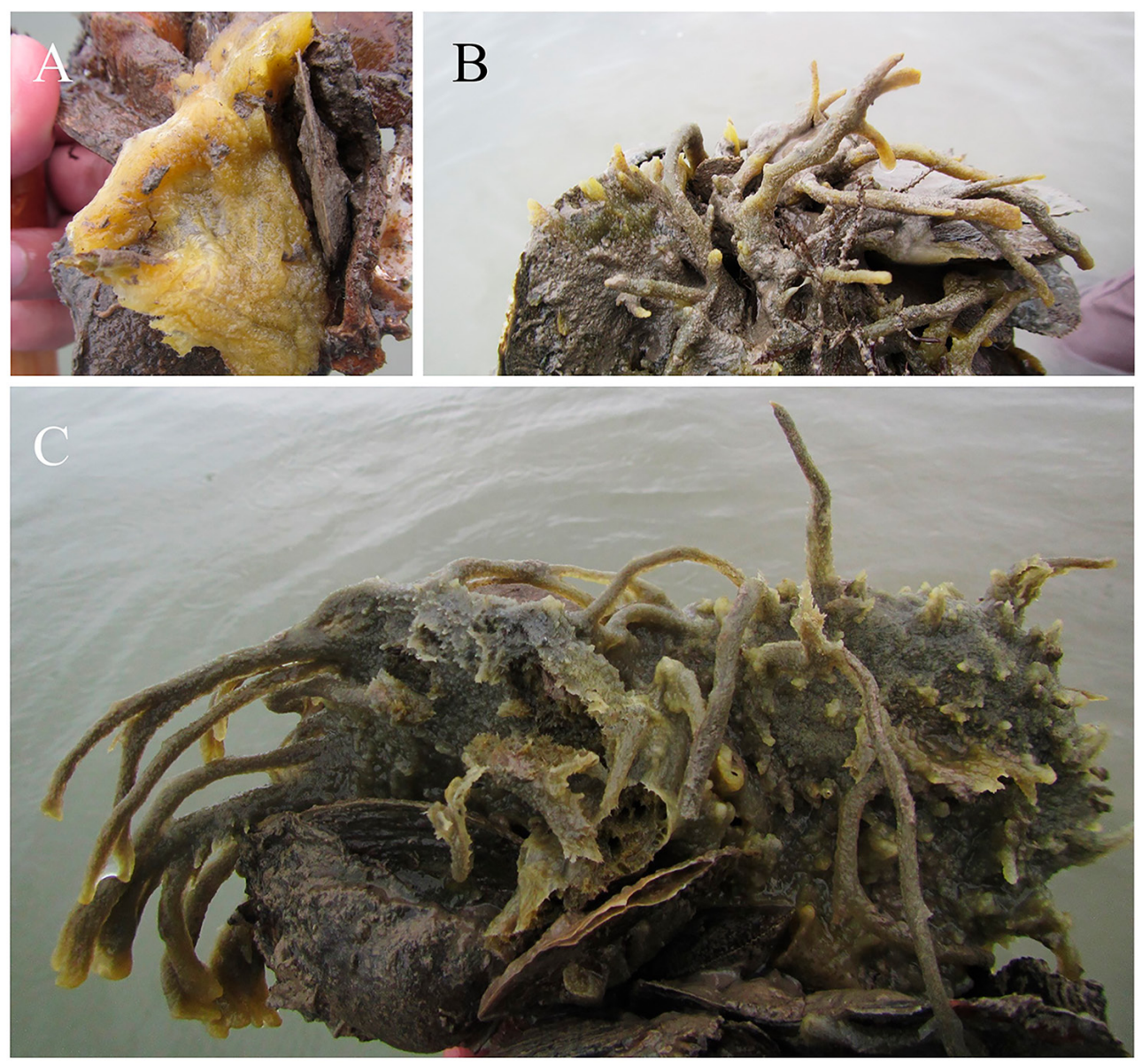
Material estudiado. CP-0001, CP-0002, CP-0003, suroeste de la laguna $\left(20.973745^{\circ} \mathrm{N},-97.340214^{\circ} \mathrm{W}\right)$, Tuxpan, Veracruz, México, $0.8 \mathrm{~m}$ de profundidad, col. V. De la Cruz (16/ IX/2015); CP-0004, CP-0005, CP-0006, CP-0007, sur de la laguna, $\left(20.976386^{\circ}\right.$ $\left.\mathrm{N},-97.343112^{\circ} \mathrm{W}\right)$, Tuxpan, Veracruz, México, $1 \mathrm{~m}$ de profundidad, col. R. G. León (02/IV/2016); CP-0008, CP-0009, suroeste de la laguna, $\left(20.983122^{\circ} \mathrm{N}\right.$, $\left.-97.346411^{\circ} \mathrm{W}\right)$, Tuxpan, Veracruz, México, $1 \mathrm{~m}$ de profundidad, col. V. De la Cruz (18/I/2019).

Descripción morfológica. Esponja incrustante ( $1.5 \mathrm{~cm}$ de espesor) a masiva con dimensiones de aproximadamente $15 \mathrm{~cm} \times 8 \mathrm{~cm}$, cubre la superficie de bivalvos (Fig. 2A). Algunos especímenes desarrollan ramificaciones prolongadas de hasta $7 \mathrm{~cm}$ de largo y $0.4 \mathrm{~cm}$ de grosor (Fig. 2B-C). Superficie rugosa y áspera al tacto. Consistencia compresible. Los especímenes en vida presentan un color amarillo y suelen estar cubiertos parcialmente por sedimento (Fig. 2C). Cuando se les preserva en alcohol adquieren un color marrón (Fig. 3A-C).
Esqueleto y espículas. Ectosoma con reticulación indefinida, las espículas se muestran dispersas (Fig. 3D-E). Coanosoma con fibras multiespiculares compuesta por oxeas grandes. Espículas meglascleras de dos tipos (Fig. 3F): oxeas (diactinas, Fig. 3G-H) y estilos (monoactinas, Fig. 3I). Oxeas ligeramente curvadas de varios tamaños (331-552.9-774 $\mu \mathrm{m} / 7-10.4$ $16 \mu \mathrm{m})$. Estilos levemente curvados $\mathrm{y}$ lisos (138-166.2-205 $\mu \mathrm{m} / 5-6.9-9 \mu \mathrm{m})$.

Distribución geográfica. Reportado previamente en Belice como Amorphinopsis sp. 1 por Rützler et al. (2000) y confirmado por Sven Zea (comunicación personal, abril 4, 2019); nororiente y suroriente de Brasil (Carvalho et al. 2004; Santos et al. 2018). Se registra por primera vez para el golfo de México.

Comentarios. Las características morfológicas externas (forma, consistencia, color) y los tipos de espículas que presentaron los especímenes estudiados, concuerdan con las descripciones de Carvalho et al. (2004) y Santos et al. (2018). Así también, sus dimensiones de las espículas se

4 Fig. 2. A. Forma incrustante de Amorphinopsis atlantica. B-C. Especímenes de A. atlantica con ramificaciones prolongadas. Laguna de Tampamachoco, Veracruz, México

Fig. 2. A. Encrusting form of Amorphinopsis atlantica. B-C- Specimens of A. atlantica with prolonged ramifications. Tampamachoco lagoon, Veracruz, Mexico 

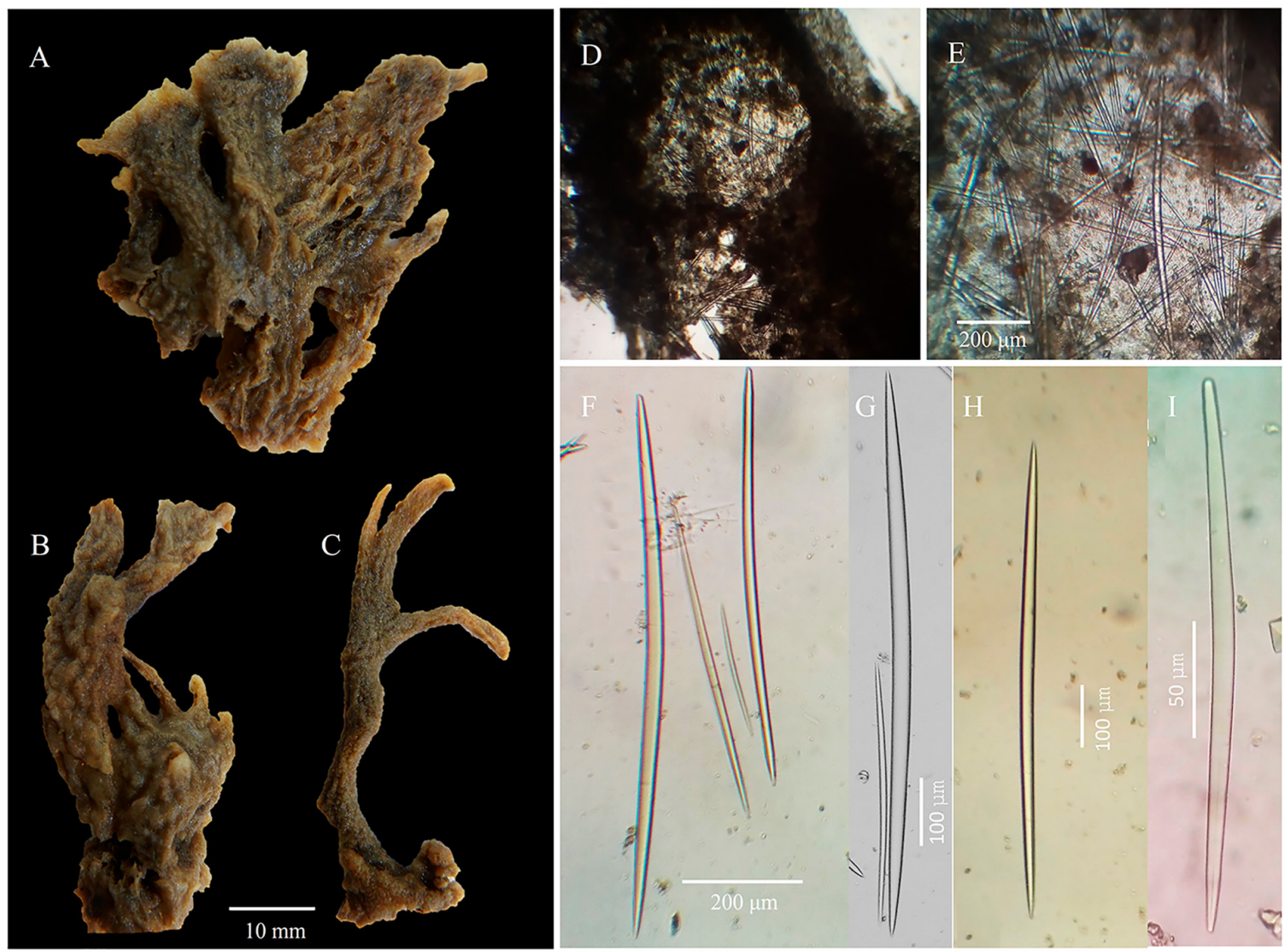

Fig. 3A-C. Forma y coloración marrón de A. atlantica preservada en alcohol etílico. D-E. Vista del esqueleto ectosomal. F-G. Espículas monoaxonicas de dos tipos: oxeas y estilos. H. Oxea lisa y ligeramente curvada. I. Estilo liso y ligeramente curvado

Fig. 3A-C. Brown coloration and form of A. atlantica preserved in ethyl alcohol. D-E. View of the ectosomal skeleton. F. Monaxon spicules of two types: oxeas and styles. H. Smooth and slightly curved oxea. I. Smooth and slightly curved style

asemejan a las medidas que se reportan en Brasil, y coincide que las oxeas tienen diferentes tamaños (Cuadro 1). Los especímenes de $A$. atlantica, se avistaron únicamente en ostras vivas de Isognomon alatus y cubiertas parcialmente de sedimento, no obstante, en Brasil, $A$. atlantica se establece en otros tipos de sustratos como en corales muertos, rocas, arena, raíces de 
mangle (Santos et al. 2018), así como también en costas rocosas entre 0.5 $16 \mathrm{~m}$ de profundidad (Carvalho et al. 2004). Como la literatura lo indica, en áreas protegidas de la luz, $A$. atlantica adopta una coloración amarillo brillante, pigmentación que fue observada en el presente estudio, lo cual se puede atribuir a la elevada turbiedad que presenta la laguna de Tampamachoco (Lara-Domínguez et al. 2011).
Se ha discutido que Amorphinopsis excavans tiene un parecido con A. atlantica, sin embargo estas especies se distinguen en el tamaño de las espículas, ya que en $A$. atlantica, la oxea más pequeña siempre es más grande que el estilo más grande, por lo que nunca se superponen (Carvalho et al. 2004), esto concuerda con las dimensiones de las espículas que se revisaron en el presente estudio. Además, $A$. excavans tiene distribución en el Indo-Pacífico, habita en arrecifes de

Cuadro 1. Valores comparativos de oxeas y estilos de Amorphinopsis atlantica. Los valores se encuentran en micras $(\mu \mathrm{m})$ ordenados de la siguiente manera: mínimo-promedio-largo máximo/mínimo-promedio-ancho máximo. $\mathrm{N}=30$ espículas

Table 1. Comparative values of oxeas and styles of Amorphinopsis atlántica. The values are in microns $(\mu \mathrm{m})$ arranged as follows: minimum-average-long maximum/minimum-average-width maximum. $\mathrm{N}=30$ spicules

\begin{tabular}{lll}
\hline Especímenes & Oxeas & Estilos \\
\hline FBUVP-00001 & $160-511.6-755 / 6-9.5-18$ & $130-146.1-180 / 6-6.2-8$ \\
FBUVP-00002 & $335-544.2-700 / 7-10.5-14$ & $127-185.2-300 / 5-7.2-8$ \\
FBUVP-00003 & $203-473.6-630 / 7-9.6-12$ & $130-142.1-159 / 5-6.3-8$ \\
FBUVP-00004 & $389-616.1-900 / 8-12.5-18$ & $138-168.2-259 / 7-7.4-8$ \\
FBUVP-00005 & $366-574.5-725 / 8-9.8-14$ & $132-140.5-159 / 5-6.9-9$ \\
FBUVP-00006 & $436-556.1-755 / 7-10.8-18$ & $130-176.1-170 / 5-6.6-10$ \\
FBUVP-00007 & $450-583.1-824 / 8-10.3-14$ & $145-152.9-172 / 6-7.1-9$ \\
FBUVP-00008 & $405-628.3-885 / 10-11.4-18$ & $167-222.9-255 / 5-7.1-10$ \\
FBUVP-00009 & $237-488.2-791 / 6-9.1-15$ & $145-161.6-192 / 5-7.8-8$ \\
Carvalho et al. (2004; holotipo & $155-392.3-825 / 8-15.5-23$ & $143-178.6-221 / 5-6.6-8$ \\
MNRJ & & \\
353), descripción original (Brasil) & & \\
Santos et al. (2018; UFPEPOR & $180-568.8-1000 / 5-15.2-31.2$ & $130-283.2-650 / 2.4-9.9-20$ \\
1666), descripción original (Brasil) & & \\
\hline
\end{tabular}


coral, desde zonas intermareales hasta $54 \mathrm{~m}$ de profundidad (Hooper et al. 1997), en cambio, A. atlantica habita en intermareales y ecosistemas estuarinos del atlántico (Carvalho et al. 2004; Santos et al. 2018).

\section{DISCUSIÓN}

El nuevo registro de Amorphinopsis se suma a los géneros Axynissa, Halichondria, Hymeniacidon, Pseudospongosorites y Topsentia, los cuales representan la familia Halichondriidae y tienen distribución en el golfo de México (Rützler et al. 2009). Sin embargo, para las costas de Veracruz únicamente se tiene el registro previo de Halichondria y Topsentia (Gómez, 2011; Ugalde et al. 2015). Por lo que, Amorphinopsis constituye el tercer género dentro del grupo Halichondriidae que se registra para Veracruz, el cual habita, especialmente en un sistema estuarino (laguna de Tampamachoco).

Las observaciones de $A$. atlantica en el área de estudio desde el 2015 a la fecha, sugieren que es una especie ya establecida en la laguna de Tampamachoco, lo cual se puede atribuir a que es tolerante a las variaciones de salinidad y sedimentación (Santos et al. 2018), variables hidrológicas que ocurren anualmente en este sistema lagunar (Lara-Domínguez et al. 2011), por ello, no se descarta que $A$. atlanti- $c a$ esté presente en otras lagunas costeras del golfo de México (aledañas, como laguna de Tamiahua y Pueblo Viejo) con características hidrológicas similares (Contreras-Espinosa, 1993).

Para finalizar, es importante destacar, que además de $A$. atlantica, otras especies de esponjas con distribución en el Caribe, se han registrado recientemente para el golfo de México. Ejemplo de ello son Mycale diversisigmata y Haliclona aff. chlorilla, observadas en el sistema arrecifal Lobos-Tuxpan (De la Cruz-Francisco \& González-González, 2016), ecosistema que colinda al occidente con la laguna de Tampachoco donde se establece precisamente $A$. atlantica.

\section{AGRADECIMIENTOS}

A los revisores anónimos por sus comentarios y sugerencias, que ayudaron a mejorar el presente documento.

\section{BIBLIOGRAFÍA}

Carvalho, M. S., Hadju, E. Mothes, B. \& Van Soest, R. (2004). Amorphinopsis (Halichondrida: Demospongiae) from the Atlantic Ocean, with the description of a new species. J. Mar. Biol. Ass. U. K., 84(5), 925-930.

Contreras, E. F. (1983). Variaciones en la hidrología y concentraciones de nutrientes del área estuarino-lagunar de Tuxpan, Tampamachoco, Veracruz, México. Biótica, 8(2), 201-213. 
Contreras-Espinosa, F. (1993). Ecosistemas costeros mexicanos. México, D. F.: Universidad Autónoma Metropolitana-Unidad Iztapalapa.

De la Cruz-Francisco V., González-González, M. \& Morales-Quijano, I. (2016). Faunística y distribución de Demospongiae: Porifera del arrecife Enmedio, Sistema Arrecifal Lobos-Tuxpan, México. CICIMAR Oceánides, 31(1), 7-16.

De la Cruz-Francisco, V. \& Bandala-Pérez, A. E. (2016). Esponjas y cnidarios (Hydrozoa y Anthozoa) del arrecife Oro Verde; cobertura bentónica y afinidad faunística con sistemas arrecifales de Veracruz, México. CICIMAR Oceánides, 31(2), 45-64.

De la Cruz-Francisco, V. \& González-González, M. (2016). Nuevos registros de esponjas para el Sistema Arrecifal Lobos-Tuxpan, con ampliaciones de ámbito de distribución para el golfo de México. Bol. Inst. Oceanogr. Venezuela, 55(2), 51-59.

De la Cruz-Francisco, V., Orduña-Medrano, R. E., Paredes-Flores, J. E., Vázquez-Estrada, R. I., González-González, M. \& Flores-Galicia, M. (2017). Una aproximación a la florística y faunística de la costa rocosa El Pulpo, Cazones, Veracruz, México. CICIMAR Oceánides, 32(1), 39-58.

Gómez, L. (2011). Esponjas marinas y de agua dulce (Porífera). En: Comisión Nacional para el conocimiento y Uso de la Biodiversidad (Conabio). La biodiversidad en Veracruz: Estudio de Estado. Comisión Nacional para el conocimiento y Uso de la Biodiversidad (pp. 217-224). Veracruz, México. Universidad Veracruzana, Instituto de Ecología, A. C.
Gómez, P. (2002). Esponjas Marinas del Golfo de México y el Caribe. México: AGT Editor, S. A.

Gómez, P. (2007). Inventario de las esponjas del Parque Nacional Sistema Arrecifal Veracruzano con nuevos registros de especies (Porifera: Demospongiae). En: A. Granados-Barba, L. Abarca-Arenas \& J.M. Vargas-Hernández (Eds.), Investigaciones cientificas en el Sistema Arrecifal Veracruzano (pp. 5172.). México: Universidad Autónoma de Campeche.

González-Gándara C., Domínguez-Barradas, C., De la Cruz-Francisco, V., SolísMarín, F. A. \& Carricart-Ganivet, J. P. (2015). Esponjas, corales escleractinios, equinodermos y peces de arrecifes coralinos del norte y sur de Veracruz. México, D. F.: SNIBCONABIO. Recuperado el 5 de enero, 2019, disponible en http://www. conabio.gob.mx/institucion/proyectos/ resultados/InfJF124.pdf

Hooper, J. N. A., Cook, S. D., Hobbs, L. J. \& Kennedy, J. A. (1997). Australian Halichondriidae (Porifera: Demospongiae): I. Species from the Beagle Gulf. In Hanley, J. R., Caswell, G., Megirian, D. \& Larson, H. K. (Eds.). Proceedings of the Sixth International Marine Biological Workshop. The marine fora and fauna of Darwin Harbour, Northern Territory, Australia (pp. 1-65). Darwin, Australia: Museums and Art Galleries of the Northern Territory and the Australian Marine Sciences Association.

Lara Domínguez, A. L., Contreras Espinosa, F., Castañeda-López, O., Barba-Macías, E.; Pérez-Hernández, M. A. (2011). Lagunas costeras y estuarios. En: Comisión Nacional para el conocimiento y Uso de la Biodiversidad (Conabio) (Eds.). La biodiversidad en Veracruz: 
Estudio de Estado (pp. 301-318). Veracruz, México. Universidad Veracruzana, Instituto de Ecología, A. C.

López-Ortega, M., Pulido-Flores, G., Serrano-Solís, A., Gaytán-Ayarzún, J. C., Monks-Sheets, W. S. \& López-Jiménez, M. A. (2012). Evaluación estacional de las variables fisicoquímicas del agua de la Laguna de Tampamachoco, Veracruz, México. UDO Agrícola, 12(3), 713-719.

Lucas M., E. \& De la Cruz-Francisco, V. (2018). Macroflora y macrofauna asociada a las raíces de Rhizophora mangle (Rhizophoraceae), en la laguna Tampamachoco, Veracruz, México. Revista Colombiana de Ciencia Animal, 10(1), 31-42.

Rützler, K., Diaz, M. C., van Soest, R. W., Zea, S., Smith, K. P., Alvarez, B. \& Wulff, J. (2000). Diversity of sponge fauna in mangrove ponds, Pelican Cays, Belize. Atoll Research Bulletin, 476, 230-248.
Santos G. G., Nascimento, E. \& Pinheiro, U. (2018). Halichondriidae Gray, 1867 from the Northeastern Brazil with description of a new species. Zootaxa, 4379(4), 556-566.

Ugalde, D., Gómez, P. \& Simões, N. (2015). Marine sponges (Porifera: Demospongiae) from the Gulf of México, new records and redescription of Erylus trisphaerus (de Laubenfels, 1953). Zootaxa, 3911(2), 151-183.

Van Soest, R. W. M., Boury-Esnault, N., Hooper, J. N. A., Rützler, K., de Voogd, N.J., Alvarez, B., ... \& Downey, R. (2018). World Porifera database. Amorphinopsis Carter, 1887. Disponible en: http:// www.marinespecies.org/porifera/porifera.php? $\mathrm{p}=$ taxdetails\&id $=131800$ on 2018-12-19. 